\title{
Intelligent Traffic Vehicles (ITV) and Road Sign Detection Systems
}

\author{
Hassanin M. Al-Barhamtoshy, Fatma M. Abdelatif and Mohamad ElBarahamtoshy \\ Faculty of Computing and Information Technology, King Abdulaziz University, Jeddah, Saudi \\ Arabia \\ hassanin@kau.edu.sa
}

\begin{abstract}
This paper monitors and detects the neighbor's activities in smart vehicle using computer vision technology and innovation of Internet of Things (IoT). These activities include vehicles monitoring, movement, and location status of vehicle. The proposed solution recognized vehicles neighbors and collects data from whole sensors of vehicle, vehicle engine, road info, mobile of the derivers, and analyze such data to make some decisions. The decision includes vehicles monitoring, automated accident, road safety, air pollution, and congestion reduction in road. Therefore, the paper suggests a data analysis method to model many devices and engines, based on ontology formulation and IoT technology. This in addition, modeling and managing over such places information from anywhere at any time. Accordingly, the current paper investigate and focus on ideation and assessing the potential of computer vision using vehicles and roads signs detections, to control traffic congestion in smart roads. Consequently, an intelligent traffic approach (vehicles and road signs recognitions) will be presented in real smart vehicles or drivers assistant system. Several scenarios to identify potential components of ITVS to recognize neighbor's vehicles based on ontology architecture and road signs issues will be analyzed and recognized. The paper sketches out and develops an ontology-based to represent vehicle, drivers, roads, traffic and weather, so five ontologies will be drawn and presented. Vehicle detection ontology-based with Haar features and computer vision library will be implemented and tested. Although, traffic detection is used to help and warn drivers for specific actions. Therefore, driving safety is increased and it will be comfort. The recognition task of the vehicles and the detection task of signs within the road will be developed and tested.
\end{abstract}

Keywords. Computer vision, vehicle system, road sing detection, intelligent traffic.

\section{Introduction}

Many prototyping technologies are used to design and implement smarts homes, cars, devices and cities. These technologies include "Grove IoT Commercial Developer Kit" with "Intel NUC System", "Intel IoT Gateway Software Suite", and the "Grove Starter Kit Plus", in addition to the "Arduino board".

Internet of Things (IoT) supports applications and services in smart homes and smart cities domains ${ }^{[1]}$. Therefore, risk examination of a smart home system designed and developed using an architecture. This architecture includes data sensors, mobile devices, in house gateway and supported tools.

One major issue present overall threat analysis in IoT architecture for security and privacy ${ }^{[2]}$. The situations of this paper illustrated private data privacy, accessibility, and safety, in a road traffic system could cause an accident.

Ziegeldorf et al. in [3] describe and examine RFID privacy with the goal of presenting relevant challenges that should 
considered in coming systems. Many of analyzed threats described, taken into their consideration the RFID based IoT systems.

Steenbruggen and et al. offered an article to manage and increase transportation safety [4]. Cheng and et al. presented a smart system to increase roads safety and traffic efficiency based on video analysis ${ }^{[5]}$.

"Vehicular ad hoc networks (VANETs)" delivers a wireless communication between moving vehicles, using a dedicated short-range communication (DSRC) ${ }^{[6]}$. Vehicles can communicate with each other's directly making "vehicle to vehicle V2V" communication or communicate with fixed equipment next to the road, denoted to as "road side unit" (RSU) creating "vehicle to infrastructure communication" (V2I) ${ }^{[7]}$.

EcoTwin $^{1}$ project announced about automating two trucks to drive closely behind each other, to approve cooperative automatic driving and to achieve safely by communicating with each other using a wireless connection. In addition, it benefits to reduce $\mathrm{CO}_{2}$ emission (through fuel consumption), improved safety (through cameras, WiFi and a radar system) and improve traffic stream. Figure 1 illustrates the smart developed car by google ${ }^{2}$. Additional study carried out that by 2040 , driverless cars will be considered by $75 \%$ of cars in the road universal.

To predict vehicle accident, an alarming smartphone system is introduced in ${ }^{[8]}$. The mobile phone application detects an accidental situation and sends alert message to the nearest emergency station. It calculates speed and variation of slant angle with accelerometer sensors and GPS in the Android mobile phone.

\footnotetext{
${ }^{1}$ https://tmc-employeneurship.com/projects/project-ecotwin-challengesemployeneurs-tno (accessed in 9/21/2017)

2 http://www.libelium.com/smart_cars_m2m_accident_prevention/ (accessed in 10/01/2017)
}

Jain and et al. presented a "smart vehicle identification system using OCR" [9]. The solution uses OCR processes technologies to recognize vehicle plate during entering and leaving the parking.

A recent paper promote and autonomous car with $80 \%$ trustworthy performance. Thus, the paper presented a smart car model-based on intelligent agents to reduce accidents ${ }^{[10]}$.

\section{Literature of Review}

Several solutions are presented for handling smart vehicles, based on sensing services. One of such solution is the reputation-aware and trajectory-based recruitment of vehicles to be used in public domains services ${ }^{[11]}$. Another case study is the data-collection presented in ${ }^{[12]}$ that requests location-aware services to ask about specific information and related location. Cases of such solution are introduced in ${ }^{[13]}$.

With the increasing of smart vehicles, an effective vehicular announcement network is established called Credit Coin ${ }^{[14]}$. Therefore, a new privacy of announcement network based on block chain and aggregation protocol is used to share traffic information.

Kate ${ }^{[15]}$ built a Block chain based in credit network solution using cryptocurrency techniques. Consequently, there is correlated effort focused on studying Block chain-based networks in a privacy-preserving way, such as Zerocash $^{[16]}$.

In other direction, road sign detection is introduced in many literatures ${ }^{[17-19]}$. Therefore, recognition process using $\mathrm{HSV}$ color space and lane contour feature to overcome the problem of finding a straight line, and line direction. In addition, a deep convolutional neural network (DCNN) is used to detect the pothole failure in roads detection. Consequently, the DCNN models have been achieved over 94\% accuracy in classifying 
images on ImageNet. The result of ${ }^{[20]}$ shows models are $96.5 \sim 97.5 \%$ in classifying a pothole in the roadway.

An overview to evaluate Internet of Things (IoT) in smart vehicles with state of art is described in ${ }^{[21]}$. Survey of wireless standard and vehicles communication modes of IoT is discussed. The survey depicts world population versus IoT devices will be 7.6 Billion people with 50 Billion devices in the IoT in 2020.

To increase efficiency of Internet of Vehicles (IoV), a "smart logistics vehicle management system" is proposed ${ }^{[22]}$. Lack of traffic information, resource wastage and transportation costs are presented.

The proposed intelligent model will be discussed in Section 3. Section 4 describes the system function and data structure with technical details. Later, Section 5 shows the implementation of the ontology domain, while as Section 6 includes features extraction in addition to result with experimental test. At the end, Section 7 discusses the conclusion and our future work.

\section{The Proposed Solution}

Many challenges are faced, due to increased population, growing size in city, and increased number of vehicles. These challenges such as: (1) Road traffic controlling and management, (2) Reducing accidents, (3) Minimizing congestion, and (4) Reducing air pollution. All these challenges will be minimized in the recent intelligent traffic system (ITS). This system is integrated from many sub-systems to deal and handle all the challenges we have.

Figure 1 describes components of Internet of Vehicles (IoV) as a context network model, in such way to support the ITVS proposed system. As shown in the figure, IoV network model is a connection network between vehicles, drivers, roads, weather, and the service provider. There are interconnections between all the network components (vehicles, drivers, roads, weather, and traffic networks) as an integration over the whole system, through the emergency service provider (ESP). ESP provides vehicles and other things objects connected to the model with they are need as services.

The proposed solution manages and controls the status of a vehicle to overcome traffic congestion and improve road efficiency. The system manager collects data from vehicles (location, speed, engine ... etc.), roads traffic, drivers' status, traffic, vehicle position relative to road, and weather. This collected data is used for data analytics and managements.

In smart vehicle, all the devices are equipped with a hybrid indoor and outdoor positions are used in tracing, motion discovery and detecting surrounding objects close to the vehicle. The aggregation of the system collect data from various sensors in the smart vehicle by convert the measurements devices into location, speed, position, steering accelerator, other vehicle nearby, road signs information.

GIS, GPS and RFID technologies are used to collect data from mobile vehicles. On the other hand, other technologies are used to control and monitor vehicle position/location, roads traffic and conditions.

The current paper design a safety system to overcome the problems of vehicle transportation, such as vehicle incidents, road security and safety, searching about vehicle, and waste of cost and time. The paper aimed to develop an overall system to observe, control and monitor all vehicle in any place and any time with suitable speed and accuracy to decrease cost, time fuel consumption and increase safety. 


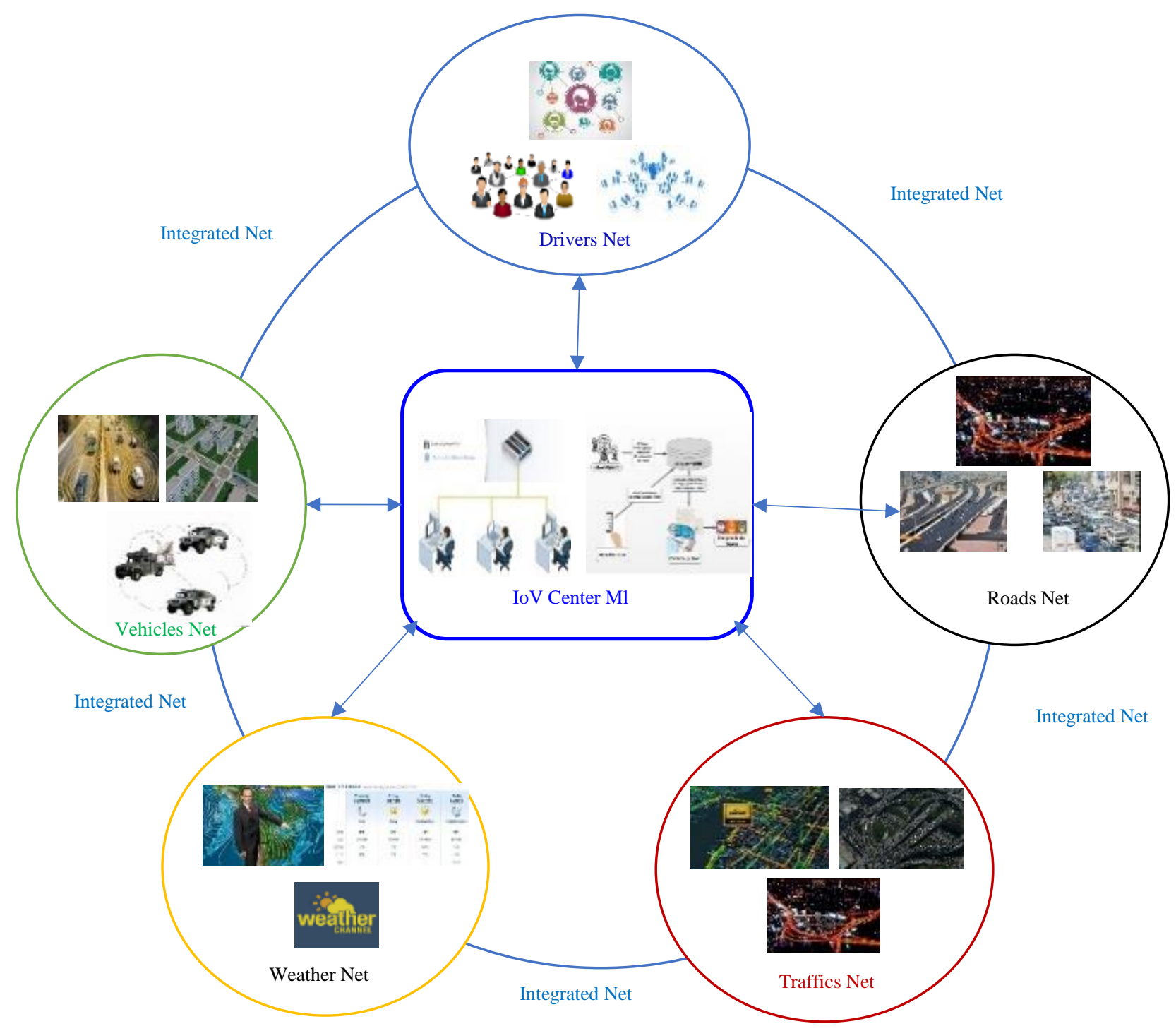

Fig. 1. Internet of Vehicles (IoV) Network Model. 


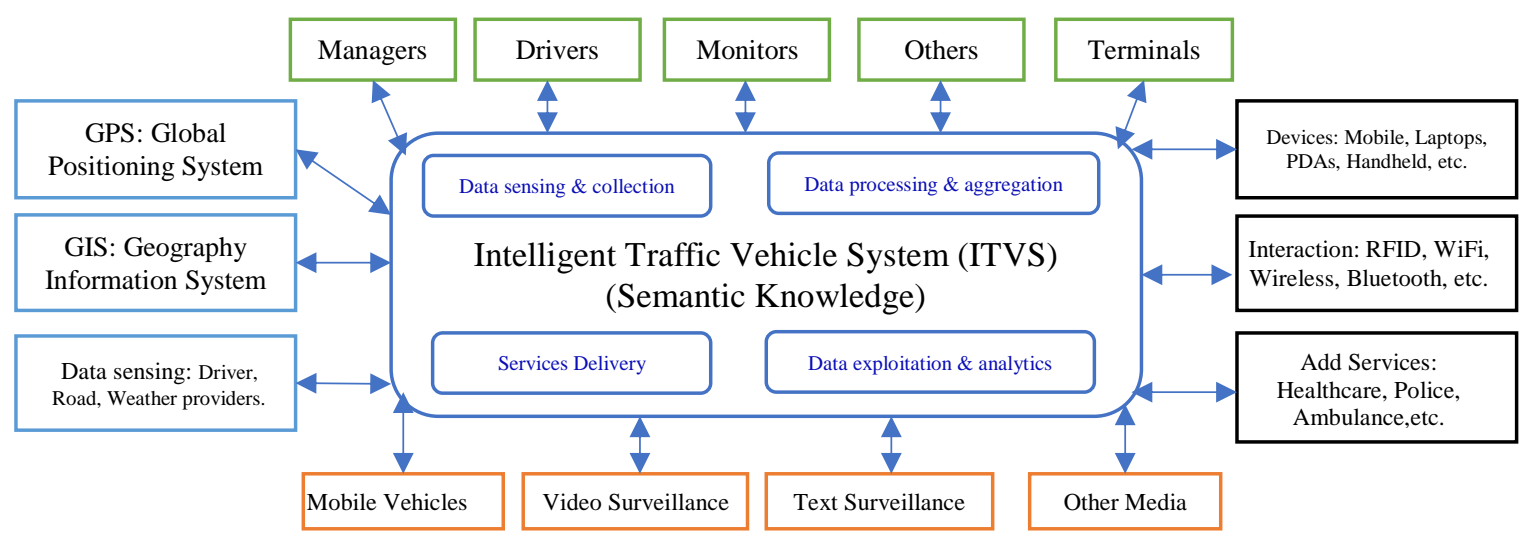

Fig. 2. The Proposed Intelligent System Architecture - from data viewpoint.

The high-level view of the system is shown in Fig. 2 as a context diagram. Five main components are described below.

1. Sensor Devices. All the devices that can be used to identify and record position, derivers characteristics/ behavior, vehicle speed, vehicle status, weather status, roads status, etc.

2. Dataset and Received Data. Such data can be classified into static and dynamic. The static data is what we call dataset that is collected before, and it is analyzed and categorized into efficient information. The dynamic data is changed over time likes object location/position; such as vehicle status, and driver behavior. The big data needs high computation power or grid computing infrastructure.

3. Interaction. Sending and receiving messages between vehicle and vehicle, driver and driver, vehicle and IT management center (managers). Most of the interaction is done through wireless communication.

4. Data

Visualization.

The representation and visualization of the information at different locations (managers, drivers, other people).

\section{Analytic}

Algorithms. The implementation of our proposed intelligent system based on dataset analytic, segmentation, classification and recognition algorithms.

Many of parameters are important: Vehicle State (c), Kinematic Processing, Vehicle Control (u), Driver Model (DMC), and Driver Assistance (if exist). The kinematic is used to calculate the physical vehicle movement as well as the deficient modeling in the driver assistance model. Based on the output of the driver assistance model a routing command $(\mathrm{u})$ is calculated by the driver model DMC in each iteration ${ }^{[23]}$.

Therefore, such calculation can be constrained with fine grain of access control to provide devices, people, or methods as needed. The access control guarantees considered rules of security and/or privacy policies. The application unit (AU) is mounted and installed into the vehicle that uses the communication capabilities of the "on board unit (OBU)" (see Fig. $3^{[6]}$ ).

\section{System Function and Data Design}

The scenario in this paper discusses security and privacy tracking to observe vehicles, drivers, roads and traffics and visualize them. Therefore, the data logger in the emergency management system allows this 
critical information needed to rescue and evacuation procedures (see Tables 1, 2, and 3).

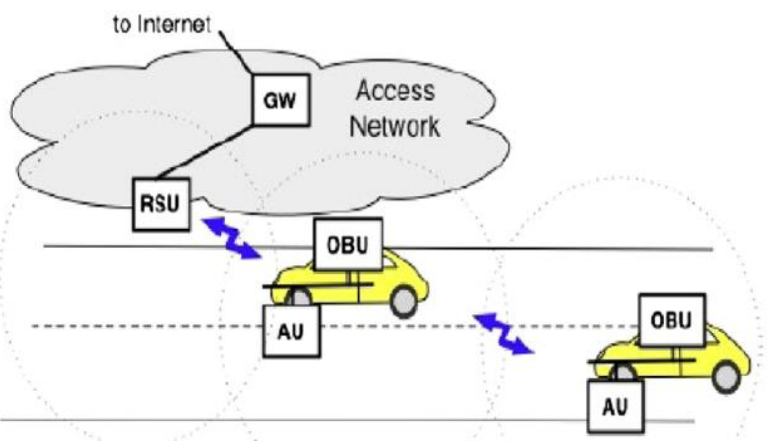

Fig. 3. RSU provides internet connectivity to the OBUs ${ }^{[6]}$.

Table 1. Vehicle Data Simulation.

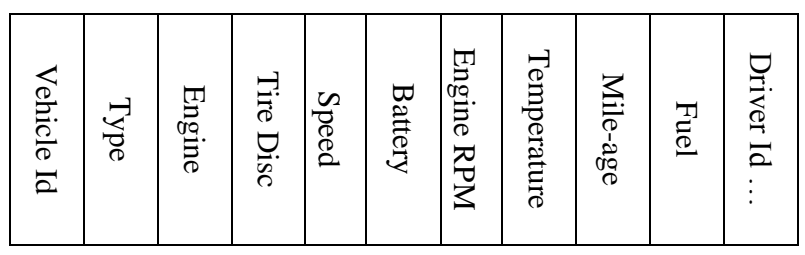

Table 2. Driver Data Simulation.

\begin{tabular}{|c|c|c|c|c|c|c|c|c|c|c|}
\hline 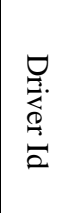 & 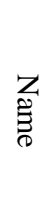 & 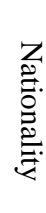 & $\begin{array}{l}\frac{\pi}{8} \\
\stackrel{D}{E} \\
\stackrel{2}{2}\end{array}$ & 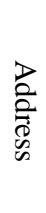 & $\begin{array}{l}\stackrel{Q}{0} \\
\stackrel{0}{0} \\
\stackrel{9}{9}\end{array}$ & 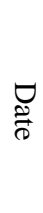 & 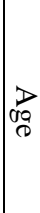 & 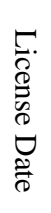 & 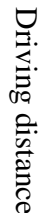 & 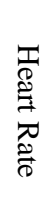 \\
\hline
\end{tabular}

Table 3. Road Data Simulation.

\begin{tabular}{|c|c|c|c|c|c|c|c|c|c|c|}
\hline $\begin{array}{l}\pi \\
0 \\
0 \\
\frac{0}{1} \\
2\end{array}$ & $\begin{array}{l}\frac{\pi}{0} \\
0 \\
\end{array}$ & $\overrightarrow{0}$ & 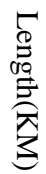 & 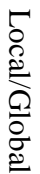 & 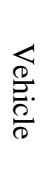 & $\begin{array}{l}\mathscr{\bigotimes} \\
\stackrel{\infty}{\infty} \\
\stackrel{\infty}{\&}\end{array}$ & $\begin{array}{l}\mathscr{n} \\
\mathbb{\infty} \\
\mathbb{8} \\
\Omega\end{array}$ & 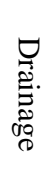 & 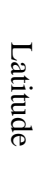 & 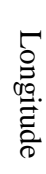 \\
\hline
\end{tabular}

Many parameters have been provided to the ITVS system, because security and privacy might be compromised. Such parameters includes road information, vehicle location, road info ( $\mathrm{Id}$, from, to, date, time), weather ...etc. These parameters are used to track driver, vehicle, and related roads activities.

Other parameters are illustrated to define roads. The roads are method to connect one point to another or built between two places, especially prepared surface that vehicles can use, see Table 3.

In an emergency-situation such as an accident during driving would have to be located with the information on the accident location, the driver and vehicle information, as well as the prior information from the management system. The effective of emergency can be increased by registered information such as: (1) which locations of accidents, (2) drivers of their historical status, and (3) vehicles prior information before accidents. Therefore, emergency service provider would have to be enabled at the accident location. Therefore, Wi-Fi access points, vehicle wireless sensors, RFID arrays, and hand-held radars are located and tracked at the accident location/position. The hand-held radar device can detect the heartbeat of a person and track movements. This radar device can differentiate between heartbeat of different people and between humans and animals at same area ${ }^{[24]}$.

\section{Ontology Concept Domain}

A concept domain integrates between vehicle, driver info, weather, traffic, and road status. Consequently, this concept domain plays semantic interoperability role in vehicle smart processing system.

The aggregator OBU cooperates between the vehicles and the infrastructure, in case of supporting driver to perform traffic flow/stream correlated activities.

The application unit (AU) (e.g. an intelligent speed adaption) can be classified into two categories: (1) Static authority, and (2) Dynamic authority. The static authority includes adding new roads, and changing in speed limitation parameters. The dynamic authority includes weather conditions, and road traffic conditions. As illustrated in Fig. 3, the on-board unit (OBU) is connected to the 
road infrastructure using GPRS interactive method. Therefore, five domain ontologies are defined here, for the aggregator OBU:

1. Vehicle ontology (V)

2. User/Driver ontology (D)

3. Road ontology (R )

4. Traffic Ontology (T)

5. Weather ontology (W)

\subsection{Ontology Definition}

During 90's, the concept of Ontology has been defined as a knowledge representation, concept description, and relation between them. Several definitions are cited to define the "ontology" term. First definition is defined by Neches et al. in ${ }^{[25]}$ : "An ontology defines the basic terms and relations comprising the vocabulary of a topic area as well as the rules for combining terms and relations to define extensions to the vocabulary". Second definition is defined by Gruber [26]: "An ontology is an explicit specification of a conceptualization". Another definition was given by Gruber: "Ontologies are defined as a formal specification of a shared conceptualization".

\subsection{Ontology Structural Design}

According to the above definitions, Fig. 4 shows the vehicle, road, driver, and sign ontological structures as context diagrams. To support the context-aware emergency services, the following infrastructure components are needed. In the road and traffic ontologies, additional information are needed such as signs and road devices, Fig. 4 (c) illustrates sign ontology. The first idea of technology car's information was published in [27] and it was supported in [28]. Hence, the current work designs the semantic concept and the ontology idea into knowledge framework. The ontology that we design in this work uses state of the art research technologies that interact with the vehicles information (Fig. 5).

\section{(A) Smart Vehicle}

The aggregator engine (on OBU, embedded in the vehicle) collects data from the indoors vehicle sensors. The smart vehicle compromises indoor tracking, motion, and sensing measurements activities into location and navigation services. The collected information from the vehicle (motion, speed, engine, ...) as the road information and mobile information (for driver or user) are sent over a secure community cloud.

\section{(B) Community Cloud}

The community cloud hosts the smart vehicle information concatenated with road and driver information. It provides access control with access restrict for integrated information. Therefore, the driver information is a part of the community cloud.

\section{(C) Emergency Service Provider (ESP)}

The emergence of ESP accesses such integrated information during normal activities services, in such way to carry out the monitoring management full processes. Figure 6 designs the ESP with smart vehicle, road information and emergency driving.

The emergency service provider (ESP) fine-grained does not required to access the smart vehicle during non-emergency condition. Coarse-grained is used in a safe mode to monitor the different activities in the ESP. Identifiers of activities vehicle location and movement will not be revealed. Whereas, during emergency, the ESP will gain access the vehicle status information with different activities.

(D) Context-aware Privacy (CAP)

Privacy and political management laws determine and participate the trust relationships with the community over the 
cloud. Relevant information will be published based on these political and law of privacy. The designed architecture provides consent of drivers, employee, and managers to be part of the privacy policy. The smart system is used to reveal, stolen car, written off or canceled vehicle, mileage data, outstanding or remaining finance, vehicle details, tax costs, and much more. At the emergency level, the ESP send the integrated service report to any assigned responders. These responders access such activity report using fine-grained information from the cloud.

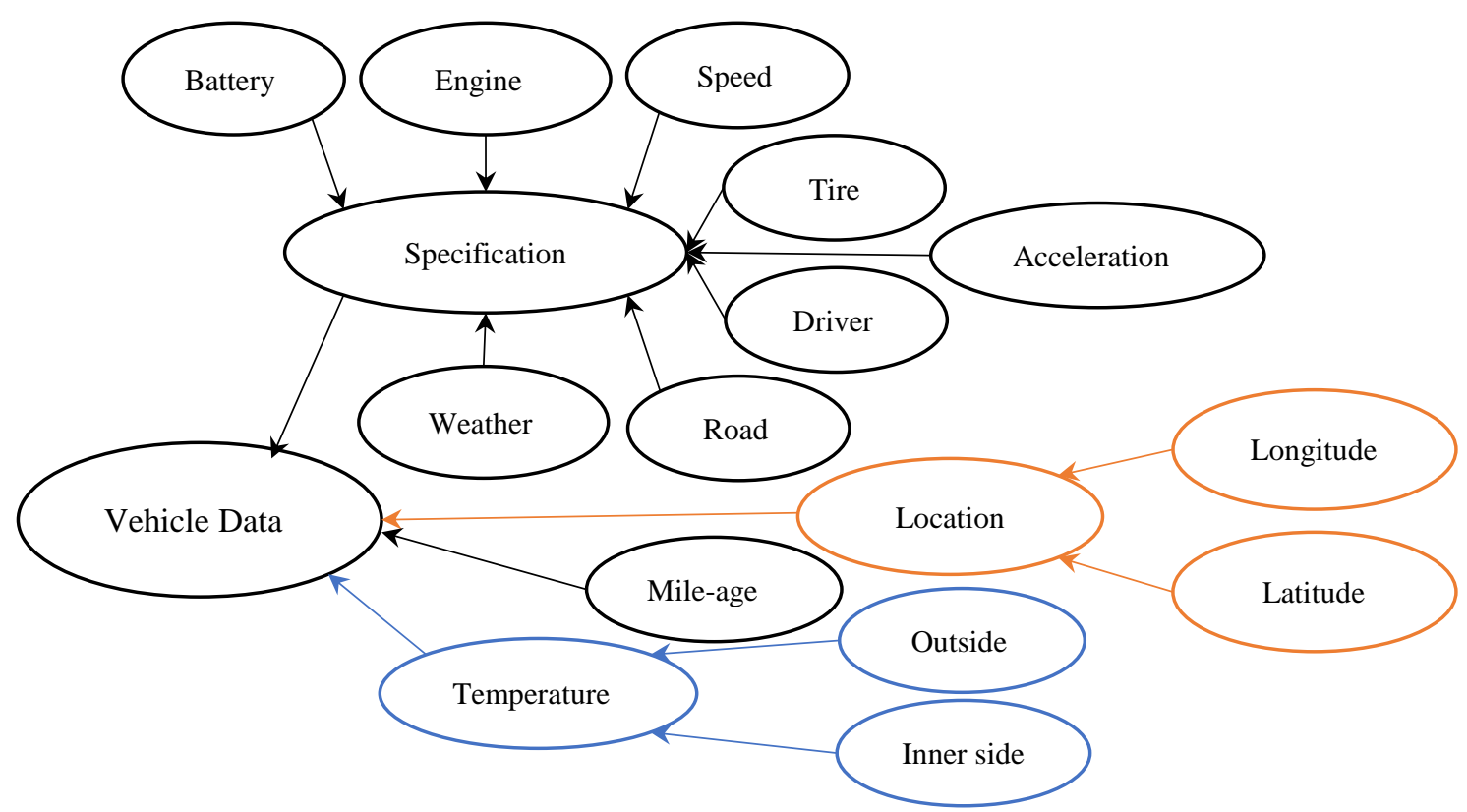

Fig. 4. (a) Vehicle Ontological Structure.

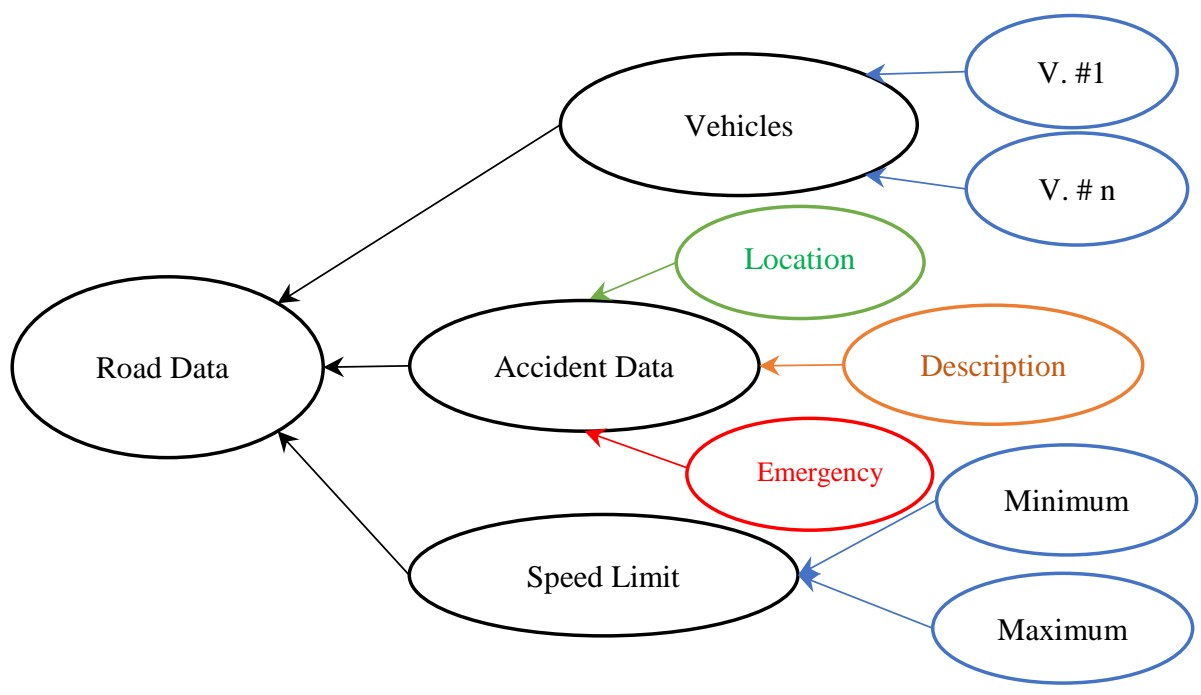

Fig. 4. (b) Road Ontological Structure. 


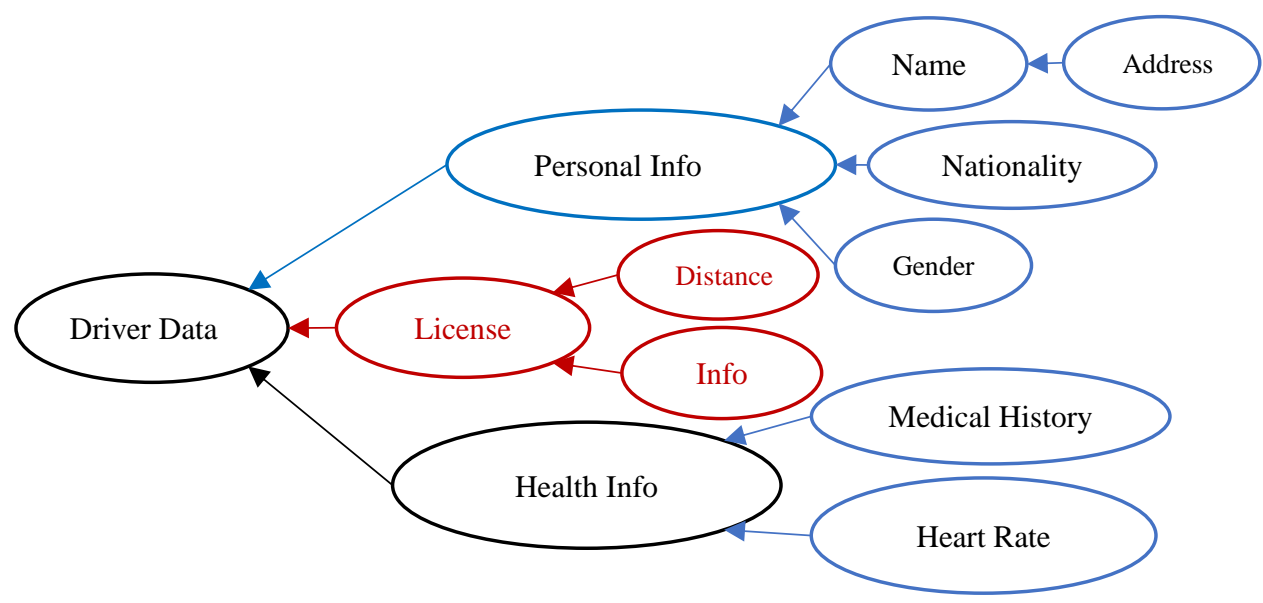

Fig. 4. (c) Driver Ontological Structure.

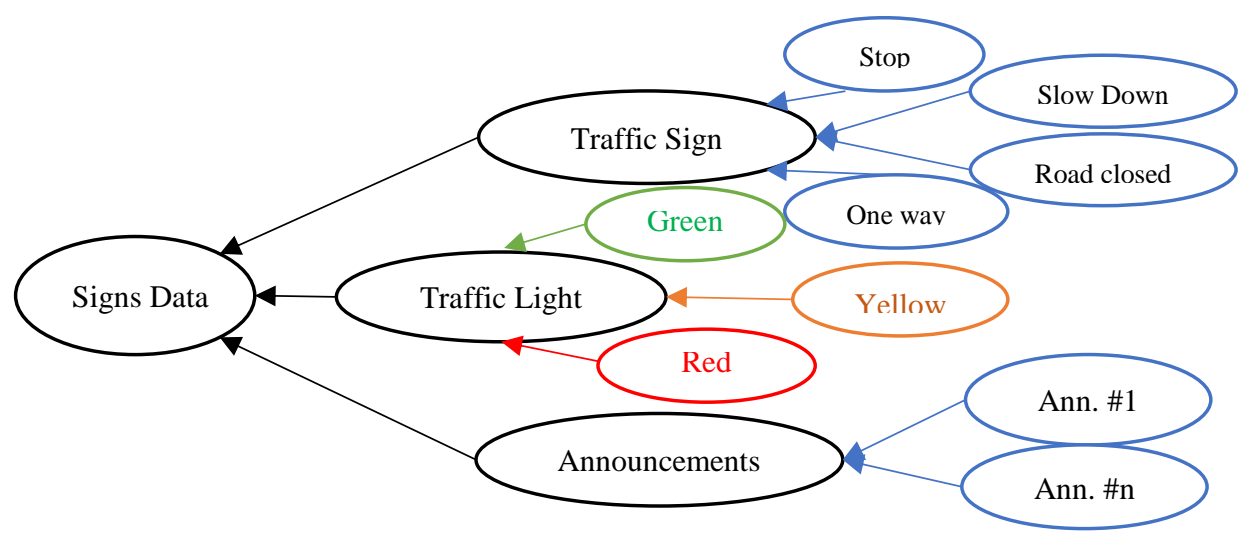

Fig. 4(d). Sign Ontology (Road ontology supporting).

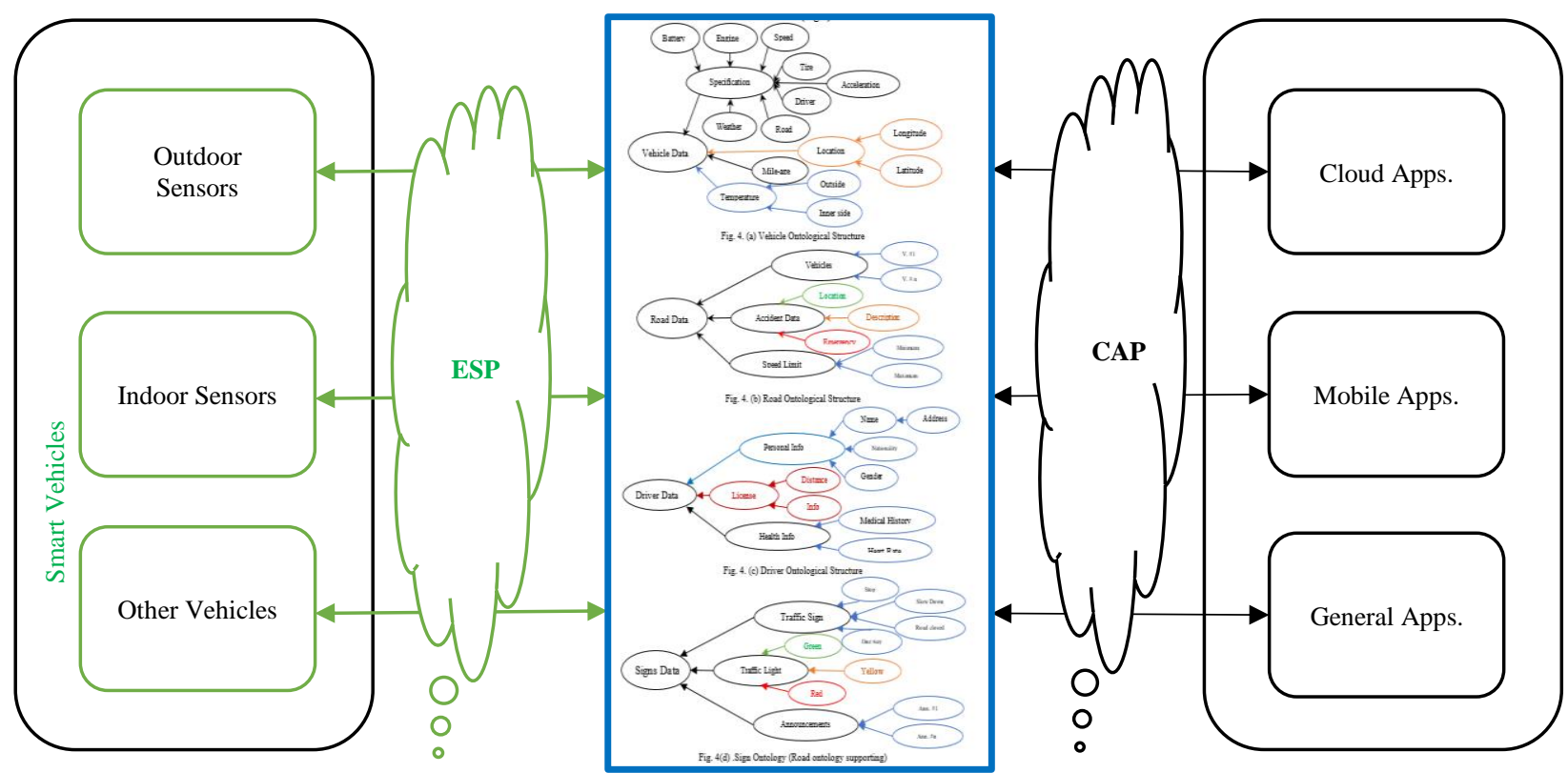

Fig. 5. The Intelligent Vehicle Traffic System (ITVS) Framework: Re-allocation between sensors and relative applications. 


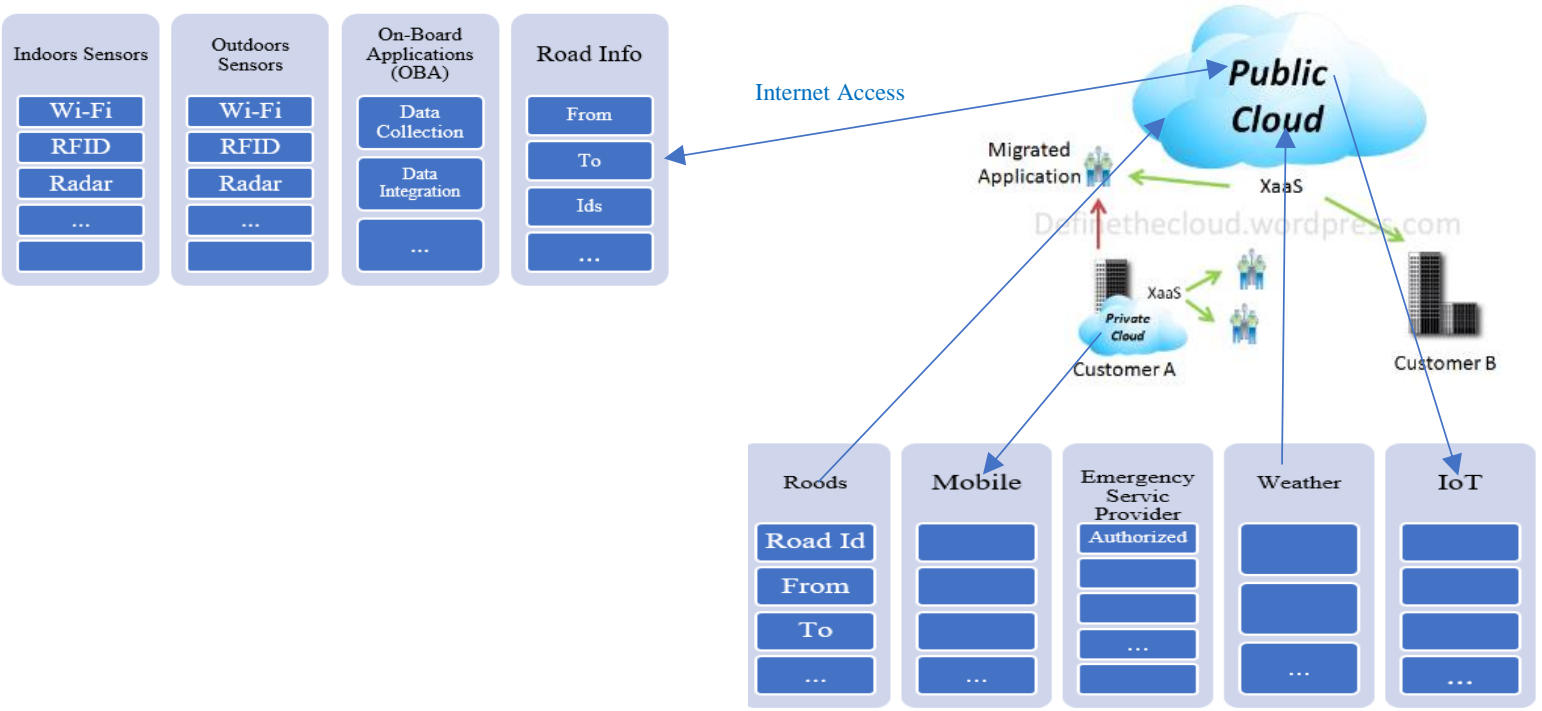

Fig. 6. Architecture of Context-Aware Emergency System.

The vehicle is collecting data from indoor and outdoor sensors, and all of this data are fed to the ITVS data center. Huge generated data for those sensors will be refined before used. Therefore, data analytics methods are refining such large data, and then store it into the cloud storage. Any data can be accessed when any connected model they are needed. So, the ITVS uses this data for processing and provide fully management processes to vehicles, and drivers. The management processes are sent to the connected indoor/outdoor and other connected objects.

The ESP receives and sends collected data from and to other modules, as well as from public and private governmental sectors and uses the ontologies of such data for analysis and refinement such data before sent to the cloud.

\section{(E) Security and Privacy}

The data collection and integration module (at the smart vehicle) send vehicle information, with a fine-grained highly sensitive awareness. The location, speed, acceleration, movement ... are calculated. The received information includes vehicle Id, vehicle info details, driver info details, and road info details. Over time, managers at management level can access such whole information to be used a history over the community cloud. Therefore, a controller program with user interface on a test-bed laptop of "Radio Control Car" (RC Car) is created and tested. Such RC Car includes battery, two DC motors (one motor at each side in addition to gear boxes to reduce speed), camera, and gas-powered due to minimum complications.

\section{Features Extraction and Testing Results}

Dataset for vehicles are collected from different resources, Fig. 7 illustrates sub samples of the used dataset ${ }^{3}$. The "Histogram of Oriented Gradients (HOG)" is used to extract features. Three main parameters are used to define size of proposed feature vector: pixel number/cell, cells number/block and orientation number. The ITVS is working according to the following steps.

Step 1. Perform a HOG to extract features, using labelled dataset.

\footnotetext{
${ }^{3}$ Samples from of California Institute of Technology dataset.
} 
Step 2. Train the classifier using linear SVM classifier.

Step 3. Implement a sliding window method to be used the trained classifier.

Step 4. Use the video stream of the road and follow detected vehicles.

Step 5. Draw a bounding box for any detected vehicle.

Our testing focused on vehicle detection and tracking using computer vision library and machine learning algorithms.

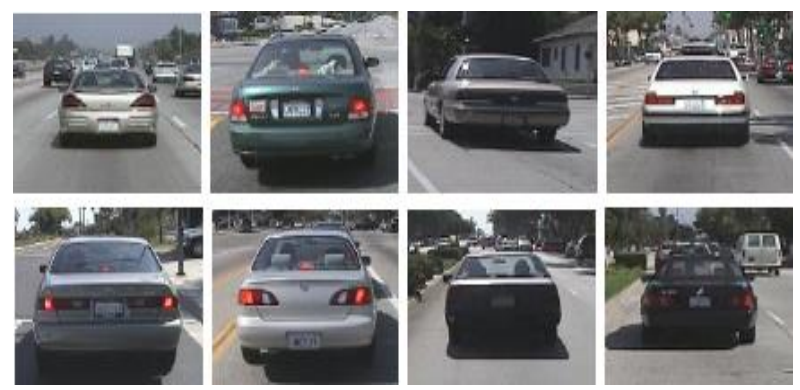

(a) California Institute of Technology sub dataset
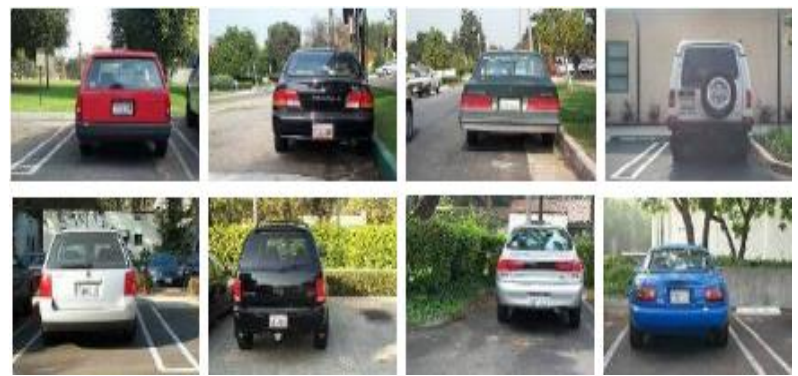

(b) Markus Weber sub samples dataset

Fig. 7. Dataset Vehicles Samples.

The first step to implement vehicle tracking is the training phase. The training phases uses dataset for vehicle images and non-vehicle images. The machine learning algorithms include a classifier to train and used to transform these images into feature spaces. Therefore, Haar cascades classifier for such testing is used. The images is extracted from vehicle dataset ${ }^{4}$. Each vehicle is described in the xml file, after applying the

${ }^{4}$ Car dataset proposed by Brad Philip and Paul Updike taken of the freeways of southern California training; this file is used to detect the vehicles (Fig. 8 shows detected seven different vehicles in (a)).

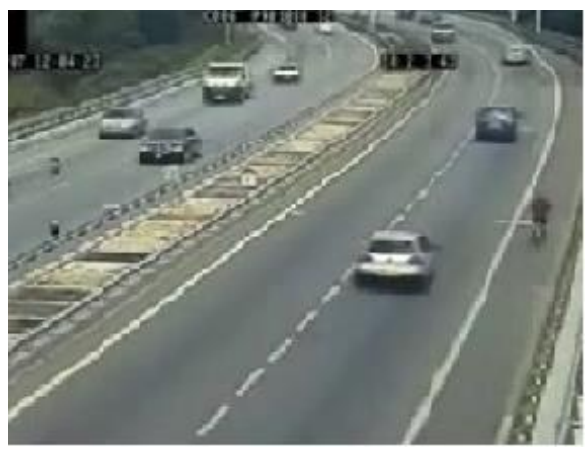

(a) Road with many objects (before detection process).

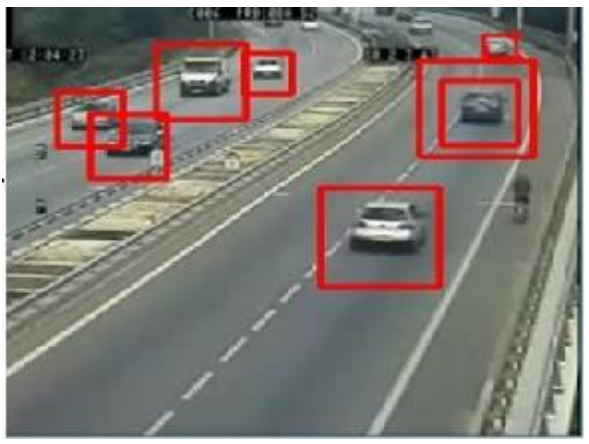

(a) Road with recognized vehicles (after detection process)

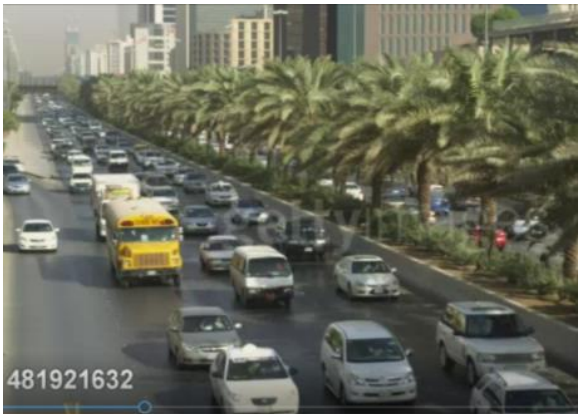

(b) Road with many objects (before detection process).

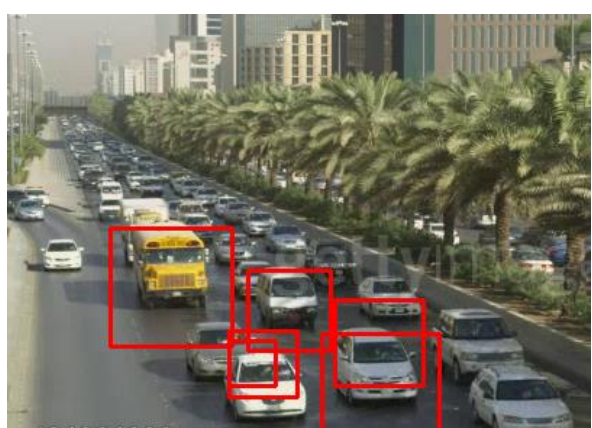

(b) Road with recognized vehicles (after detection process) 


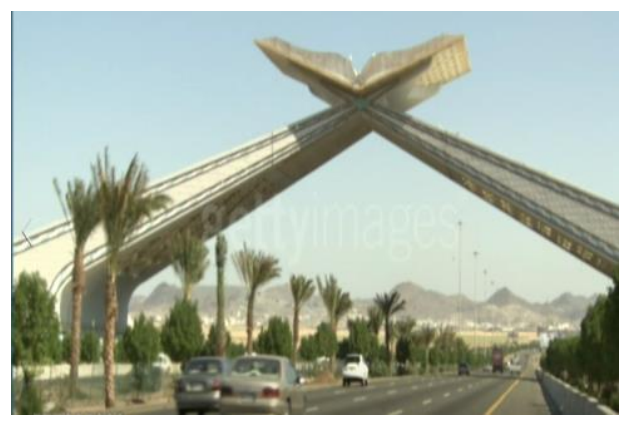

(c) Road with many objects (before detection process).

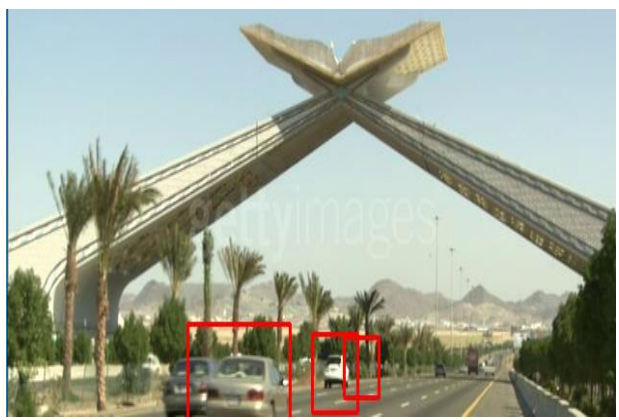

(c) Road with recognized vehicles (after detection process)

Fig. 8. The Vehicles detection process.

Traffic sign recognition (TSR) is an important research topic in the computer vision field. Many of competition have been done, "German Traffic Sign Recognition Benchmark (GTSRB)" in $2011^{\text {[29] }}$. There is no fully standard for all traffic signs over the world. The United States signs are significantly different from those in Europe countries, according to the international variations in traffic sign designs. TSR now is relevant and driving becomes more and more automated. There are traffic datasets such as German traffic sign detection GTSD and Belgium traffic sign detection BTSD ${ }^{[30]}$.

\subsection{Traffic Detection}

Traffic stream is varying over road capability, road space and time. In practice, the traffic characteristics parameters are flow rate direction, vehicles speed, road congestion, video stream clearing, stream timing (nighttime or daytime).
The flow rate (f) is defined as the number of counted vehicles $(\mathrm{N})$, divided by duration time $(\mathrm{T})$.

$$
f=\frac{N}{T}=\frac{1}{\frac{1}{N} \sum_{i} h_{i}}=\frac{1}{h}
$$

Therefore, to be able to identify the vehicles in meaningful way, there is standard recorded time (limit) for road traffic headways $(h)$. Also, the speed is required to observe recognize and control for vehicles. To create the speed criteria, it need distance space and time. The speed of an individual vehicle is calculated by:

$$
V_{i}=\frac{d x}{d y}=\lim _{t_{2}-t_{1} \rightarrow 0} \frac{x_{2}-x_{1}}{t_{2}-t_{1}}
$$

Therefore, to calculate the average speed, the following criteria is used:

$$
\bar{V}=\frac{1}{N} \sum_{i=1}^{N} V_{i}
$$

Where $V_{i}$ represents mean or average speed. Also, the average speed $\left(\widetilde{V}_{l}\right)$ can be defined as a cross distance divided by average time.

$$
\tilde{V}_{i}=\frac{D}{\frac{1}{N} \sum_{i} t_{i}}
$$

Where $t_{i}$ represents time for vehicle $\mathrm{i}$ to cross distance D.

$$
t_{i}=\frac{D}{V_{i}}
$$

Therefore, if the vehicle crossed the full distance (D) within duration time (t), it can be recognized. We assume that there is no completely overlapping between vehicles flow to simulate estimated density convergence. In this topic, the average number of recognized vehicles depends on road and traffic congestion. According to the status of the road and traffic condition, the accuracy of the ITVS proposed system will be affected. Figures 8(a), (b), and (c) illustrate detailed testing in different roads, locations, and congestions. 


\subsection{Sign Detection}

In order to test and evaluate the ITVS solution, vehicle and traffic dataset are needed. The "LISA traffic" as a vehicle dataset and TSR dataset as a sign dataset [31] are downloaded and used. Usually, flow rates headways $(h)$ is expressed in terms of vehicles/hour.

The second implemented case study here in the paper is the "stop sign" recognition. The test bed of the proposed prototype of the "stop sign" depends on mean square error computations. The used dataset of this testing composed from 100 signs $^{5}$. Figure 9 illustrates the recognition process (green rectangular) with the accuracy and execution of the recognition time of such signs in different locations. If the images are not including any "stop sign", the proposed solution reply by "'None Type' object has no attribute with ' Stop sign shape"'.

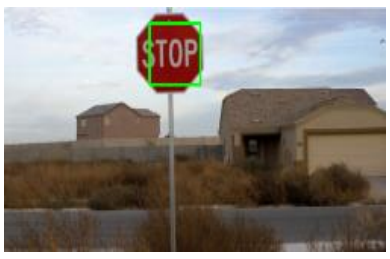

(a) Execution time $=25.40$ sec; Accuracy $=97.30 \%$

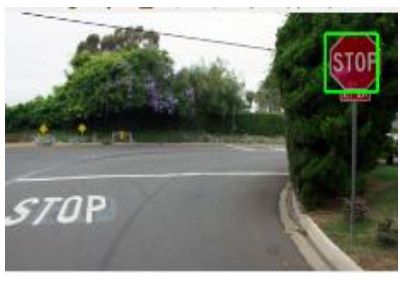

(d) Execution time $=12.99$ sec; Accuracy $=98.30 \%$

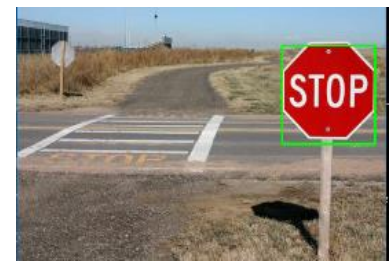

(b) Execution time $=27.53$ sec; Accuracy $=93.35 \%$

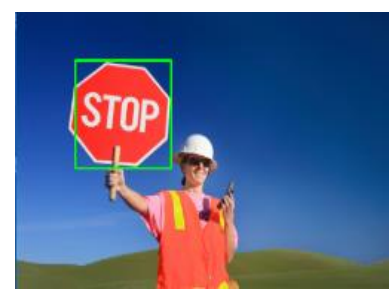

(e) Execution time $=29.58$ sec; Accuracy $=95.0 \%$
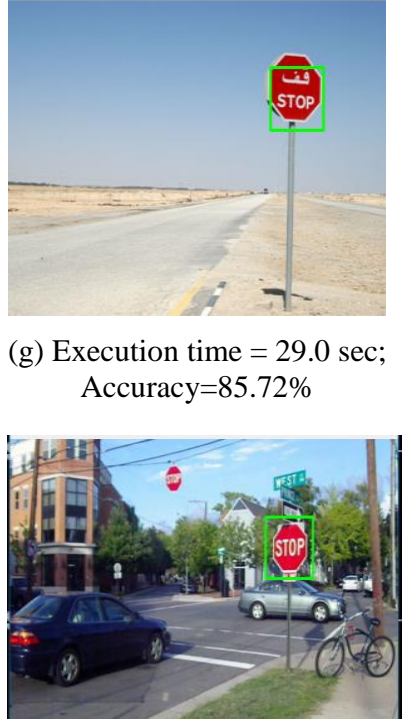

(i) Execution time $=29.0 \mathrm{sec}$; Accuracy $=84.85 \%$

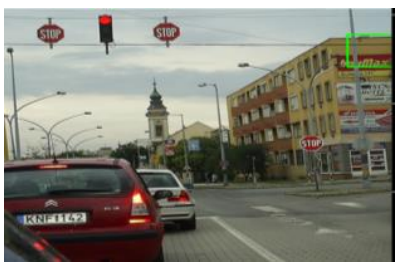

(k) Execution time $=29.0 \mathrm{sec}$; Accuracy $=86.61 \%$ (g) Execution time $=29.0 \mathrm{sec}$; Accuracy $=85.72 \%$

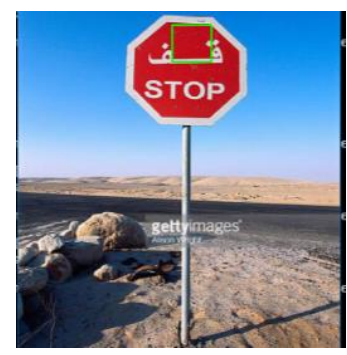

(h) Execution time $=19.43$ sec; Accuracy $=84.0 \%$

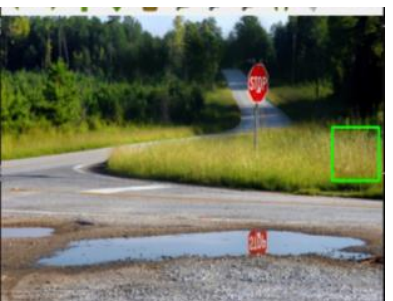

(j) Execution time $=27.92$ sec; Accuracy $=91.70 \%$

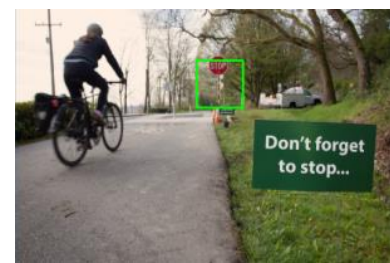

(1) Execution time $=30.74$ sec; Accuracy $=81.12 \%$
The processing speed was 25.2 second, whereas the average accuracy of recognition was $90.14 \%$.

Fig. 9. The Stop Signs detection processes.

The ITVS accuracy depends on video streaming quality of the road (obstacles, shadow from surrounding objects). The ITVS algorithms recognize such empty road as obstacles caused from weather condition. Weather status change over time ${ }^{[32]}$ (rainfall, clouding, sunshine, shadow trees, and transparency).

Note that, referring to Fig. 9(j), the recognized STOP sign (green rectangular) is located in average location between the original sign and the mirror sign (in the drainage water). Also, within the recognized STOP sign in the case of Fig. $9(\mathrm{k})$, is displayed at wrong location due to the doubling of the STOP sign. 


\section{Conclusion and Future Work}

This paper introduced a smart vehicle threat solution, and analyzed it in our test bed design. Our initial analysis demonstrates that existing smart vehicle using IoT infrastructure is managed to get access to the underlying network. The paper discussed to semantic and ontology representation of vehicle, road, road's sign, and weather related descriptions. The ontology concept provided a semantic modeling for interaction between different frameworks. The unified representation of the ontology: vehicle, driver, road, weather and traffic are used to capture features in order to monitor, manage, and decide security and privacy issues. Therefore, the ontology definition supports the specification and implementation phases for smart vehicle interaction. Vehicle tracking sub-system is tested based on computer vision library, Haar classification and ontology modeling.

During the recognition phase of the ITVS, two main tasks are done; vehicles detections and signs recognitions. An intelligent traffic approach is presented in real smart vehicles model or drivers assistant system. Both detection and recognition showing us a promising results. Therefore, traffic signs detection were performed to recognize the road sign patterns in case of facility driving by fully automation of the intelligent vehicle. Furthermore, natural background of roads, weather conditions, unclear signs, and air population remain challenged tasks.

Now, the ITVS assists to inspect roads cases, accidents, vehicles status, environmental operation for drivers, ambient weather and air quality, and finally, vehicle management system.

In future, we are scheduling to extend our proposed solution to demonstrate in detail the significances and the impact of the improvement of vehicle performance with deriver efficiency.

\section{References}

[1] Geneiatakis, D., Kounelis, I., Neisse, R., Nai-Fovino, I., Steri, G. and Baldini, G. (2017). Security and Privacy Issues for an IoT based Smart Home, MIPRO 2017, May 22- 26, 2017, Opatija, Croatia, pp: 1292-1297.

[2] Kozlov, D., Veijalainen, J. and Ali, Y. (2012). "Security and Privacy Threats in IoT Architectures," In: Proceedings of the 7th International Conference on Body Area Networks, ser. BodyNets '12. ICST, Brussels, Belgium, Belgium: ICST (Institute for Computer Sciences, Social-Informatics and Telecommunications Engineering), 2012, pp: 256-262.

[3] Ziegeldorf, J., Morchon, O. and Wehrle, K. (2014). "Privacy in the internet of things: threats and challenges," Security and Communication Networks, 7, (12): 27282742, 2014. Available: http://dx.doi.org/10.1002/sec.795.

[4] Steenbruggen, J., Borzacchiello, M., Nijkampa, P. and Scholten, H. (2013). Data from telecommunication networks for incident management: An exploratory review on transport safety and security. Transport Policy, 28: 86102. doi:10.1016/j.tranpol.2012.08.006

[5] Cheng, H., Gau, V., Huang, C. and Hwang, J. (2012). Advanced formation and delivery of traffic information in intelligent transportation systems. Expert Systems with Applications, 39(9): 8356-8368. doi:10.1016/j.eswa. 2012.01.184

[6] Al-Sultan, S., Al-Doori, M., Al-Bayatti, A. and Zedan, H. (2013). A comprehensive survey on vehicular Ad Hoc network, Journal of Network and Computer Applications, Elsevier, http://dx.doi.org/10.1016/j.jnca.2013.02.036

[7] Moustafa, H. and Zhang, Y. (2009). Vehicular networks: techniques, standards, and applications, CRC Press.

[8] Faiz, A., Bin Imteaj, A. and Chowdhury, M. (2015). Smart Vehicle Accident Detection and Alarming System Using a Smartphone, 1st International Conference on Computer \& Information Engineering, 26-27 November, 2015, Rajshahi, Bangladesh.

[9] Jain, K., Choudhury, T. and Kashyap, N. (2017). Smart Vehicle Identification System using OCR, 3rd IEEE International Conference on "Computational Intelligence and Communication Technology" (IEEE-CICT 2017).

[10] Bourbakis, N. Alamaniotis, M. and Tsoukalas, L. (2017). A Smart Car Model based on Autonomous Intelligent Agents for Reducing Accidents, 2017 IEEE Transportation Electrification Conference and Expo (ITEC), pp: 767 - 772.

[11] Abdelhamid, S., Hassanein, H. and Takahara, G. (2018). Reputation-Aware, Trajectory-Based Recruitment of Smart Vehicles for Public Sensing, IEEE 
Transactions on Intelligent Transportation Systems, 19(5) May 2018: 1387- 1400.

[12] Palazzi, C. E., Pezzoni, F. and Ruiz, P. M. (2012). "Delay-bounded data gathering in urban vehicular sensor networks," Pervasive Mobile Comput., 8(2): 180193.

[13] Lee, U. and Gerla, M. (2010). "A survey of urban vehicular sensing platforms," Comput. Netw., 54(4): $527-544$.

[14] Li , L., Liu, J., Cheng, L., Qiu, S., Wang, W., Zhang, X. and Zhang, Z. (2018). CreditCoin: A PrivacyPreserving Blockchain-Based Incentive Announcement Network for Communications of Smart Vehicles, IEEE Transactions on Intelligent Transportation Systems, 19(5) May 2018: 1- 17.

[15] Kate, A. (2016). "Introduction to credit networks: Security, privacy, and applications," In: Proc. ACM SIGSAC Conf. Comput. Commun. Secur., Oct. 2016, pp: 1859-1860.

[16] Garman, C., Green, M. and Miers, I. (2016). Accountable Privacy for Decentralized Anonymous Payments. IACR Cryptology ePrint Archive. [Online]. Available: http://eprint.iacr.org/2016/061.

[17] Kortli, Y., Marzougui, M., Bouallegue, B., Subash, J., Bose, Chandra, Rodrigues, P. and Atri, M. (2017) "A Novel Illumination-Invariant Lane Detection System", ICACC2017 Conf., pp. 166-171. Mar. 2017.

[18] Zuo-Quan, Li, Hui-Min, Ma and Zheng-Yu, Liu (2016) "Road Lane Detection With Gabor filters", ISAI2016 Conf., pp. 436-440. June 2016.

[19] Kim, J., Kim, S., Lee, S., Lee, T. and Lim, J. (2018). Lane Recognition Algorithm using Lane Shape and Color Features for Vehicle Black Box, 2018 International Conference on Electronics, Information, and Communication (ICEIC), pp. 1-2.

[20] An, K., Lee, S. Ryu, S. and Seo, D. (2018). Detecting a pothole using Deep Convolutional Neural Network models for an adaptive shock observing in a vehicle driving, 2018 IEEE International Conference on Consumer Electronics (ICCE), pp. 1-2.

[21] Keertikumar, M., Shubham, M. and Bankar, R. (2015). Evolution IoT in Smart Vehicles: An Overview, 2015 International Confrence on Green Computing and Internet of Things (ICGCIoT), pp. 804-809.

[22] Sharma, N., Ghauhan, N. and Chand, N. (2016). Smart Logistics Vehicle Management System based on Internet of Vehicles, 2016 Fourth International Conference on
Parallel, Distributed and Grid Computing (PDGC), pp. 495-499.

[23] Fuchs, C., Knopp, B., Z*obel, D. and Paulus, D. (2017). Model-based Evaluation of Practical Sensor Noise Impacts in Articulated Vehicle Driving Scenarios, 2017 IEEE Intelligent Vehicles Symposium (IV), June 11-14, 2017, Redondo Beach, CA, USA

[24] Srinivasan, R., Mohan, A. and Srinivasan, P. (2016). Privacy Conscious Architecture for Improving Emergency Response in Smart Cities, Smart City Security and Privacy Workshop (SCSP-W), IEEE 2016.

[25] Neches, R., Fikes, R. and Gruber, T. R. (1991). Enabling Technology for Knowledge Sharing. AI Magazine, 12(3): 36-56.

[26] Gruber, T. R. (1995). Towards Principles for the Design of Ontologies Used for Knowledge Sharing. International Journal of Human-Computer Studies, 43: 907-928.

[27] Feld, M. and Endres, C. (2010). Sharing User and Context Models in Automotive HMI. In: Adjunct Proceedings of the Second International Conference on Automotive User Interfaces and Interactive Vehicular Applications, Pittsburgh, United States, November 2010.

[28] Feld, M. and Müller, C. (2011). The Automotive Ontology: Managing Knowledge inside the Vehicle and Sharing it Between Cars, Automotive UI '11, Proceedings of the 3rd International Conference on Automotive User Interfaces and Interactive Vehicular Applications, pp: 79-86, ACM.

[29] Møgelmose, A., Liu, D. and Trivedi, M. (2015) Detection of US Traffic Signs, IEEE Transactions on Intelligent Transportation Systems, 16(6): 3116-3125.

[30] Hossain, S. and Hyder, Z. (2015). Traffic Road Sign Detection and Recognition for Automotive Vehicles, International Journal of Computer Applications, 120(24) June 2015.

[31] Belaroussi, R., Foucher, P., Tarel, J., Soheilian, B., Charbonnier, P. and Paparoditis, N. (2015). Road Sign Detection in Images: A Case Study. In: Proceedings of the 20th International Conference on Pattern Recognition (ICPR), Istanbul, Turkey, 23-26 August 2010; pp. 484-488.

[32] McHugh, D. (2014). Traffic Prediction and Analysis using a Big Data and Visualization Approach, Master Thesis in Computer Science, Business Intelligence and Data Mining, Institute of Technology Blanchardstown, Ireland, September 2014. 


\section{نظم المركبات المرورية الذكية ونظم التعرف على إثارات الطرق حسنين البرهمتوشي، و فاطمة عبد اللطيف، و محمد البرهمتوشي}

قسم تقنية المعلومات، كلية الحاسبات وتقنية المعلومات، جامعة الملك عبد العزيز، جدة، المدلكة العربية

$$
\text { السعودية }
$$

hassanin@kau.edu.sa

الدستخلص. تقدم هذه الورقة طريقة للتعرف على الأنثطة والأحداث القريبة من السيارة باستخدام

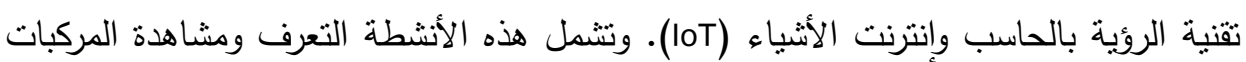

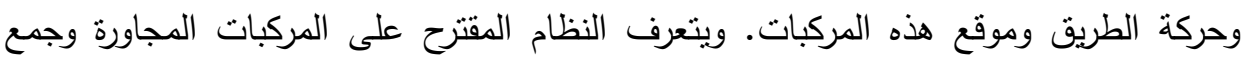

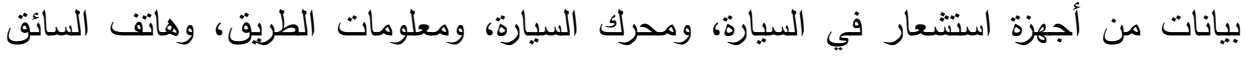
(الجوال)، وتحليل هذه البيانات لاتخاذ بعض القرارات. وتشمل الأنشطة مراقبة هذه المركبات، وسلامة الطريق، وتلوث الهواء، والدد من الازدحام في الطرق. لذلك، نقتزح هذه الورقة طريقة

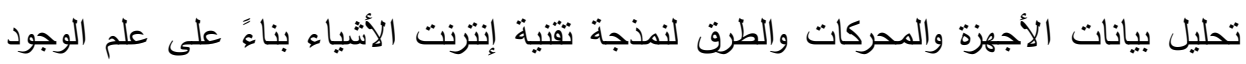
. Ontology على ذلك، تقوم الورقة بالتحقيق والتركيز على التفكير وتقييم إمكانات الروئية بالحاسب باستخدام

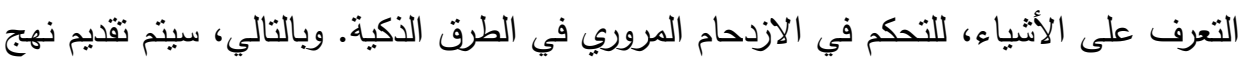

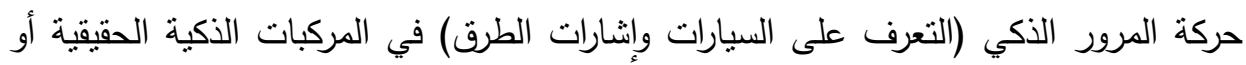

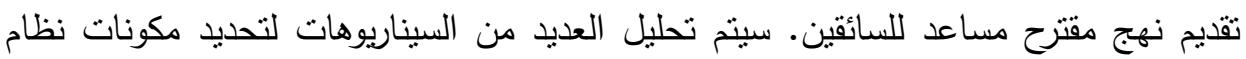
ITVS

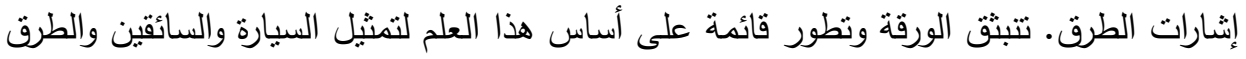
وحركة المرور والطقس، بحيث يتم رسم نمذجة الخمسة أنماط. سيتم بناء وتتفيذ واختبار النظام

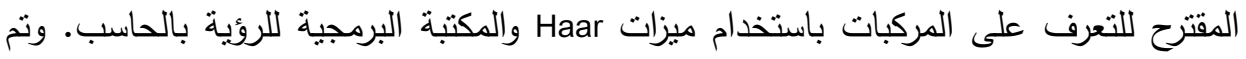
نطوير واختبار التعرف على المركبات ومهمة الكثف والتعرف عن علامات الطريق بكفاءة عالية. الكلمات الدفتاحية: روية بالحاسب، نظام السيارة، كثف علامات الطريق، حركة المرور الذكية. 